\title{
Developing and validating a media health literacy scale with a critical approach for high school students in Tehran
}

\author{
Abolghasem Raeisi Nafchi ${ }^{1}$, Abolfazl Danaei ${ }^{1 *}$, Seyed Mohammad Zargar ${ }^{1}$ \\ 1. Department of Management Media, Semnan Branch, Islamic Azad University, Semnan, Iran
}

Received: 19 October 2020

Accepted for publication: 5 September 2021

[EPub a head of print-13 September 2021]

Payesh: 2021; 20 (5): 609- 617

\begin{abstract}
Objective(s): Every society has a level of media literacy, but what is important is a critical approach to media content, which is of particular importance among adolescents and young people. Therefore, in this study, a model for media health literacy with a critical approach for high school students in Tehran has been developed and validated.

Methods: The research method in this article is of quantitative type. The statistical population of this study includes the first and second year high school students of Tehran. The statistical sample was based on the sample required for exploratory or confirmatory factor analysis of 415 persons (in two stage) and the cluster sampling method was proportional to the selected cluster size. The research instrument was a self-designed questionnaire, which was validated from the exploratory and confirmatory factor analysis using SPSS and PLS software for data analysis.

Results: The findings for the exploratory factor analysis showed that the factor loadings of all indicators were higher than 0.3 . The goodness indicators of the pattern fit also showed that the structural pattern extracted has a good fit with the data. Also, the dimensions and components of the model had good reliability (Cronbach's alpha and combined reliability).

Conclusions: The findings suggest that the need for integrated and systematic attention to all aspects of media health literacy with a critical approach in high schools is necessary.
\end{abstract}

Key words: Media literacy, health literacy, critical approach

\footnotetext{
* Corresponding author: Semnan Branch, Islamic Azad University, Semnan, Iran

E-mail: a.danaei@semnaniau.ac.ir
} 


\title{
تدوين و اعتباريابى ابزار سواد سلامت رسانه اى با رويكرد انتقادى براى دانش آموزان دوره متوسطه دوم
}

\author{
ابوالقاسم رئيسى نافجى ' ، ابوالفضل دانايى '، سيد محمد زركر' \\ 1. كروه مديريت رسانه، واحد سمنان، دانشخاه آزاد اسلامى، سمنان، ايران

جـكيده

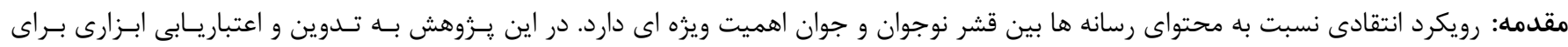

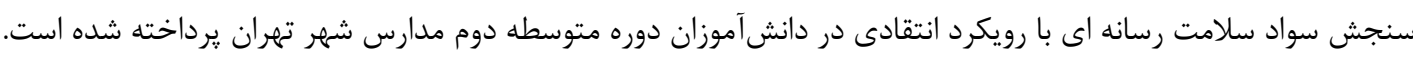

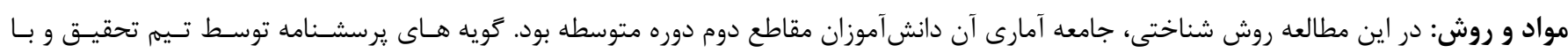

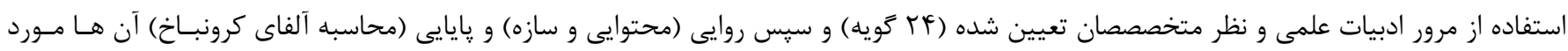

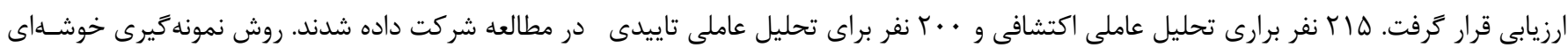
متناسب با حجم خوشه بود.

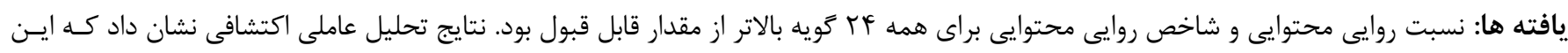

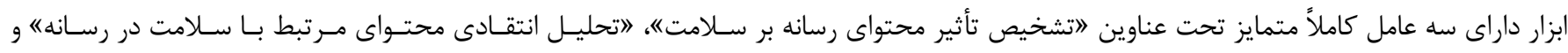

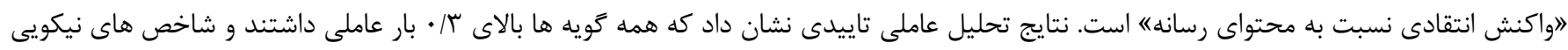

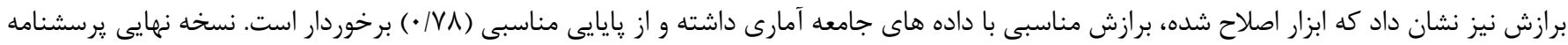

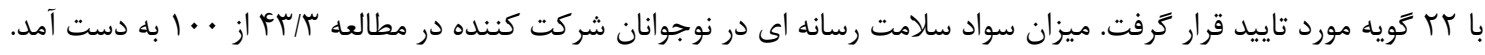

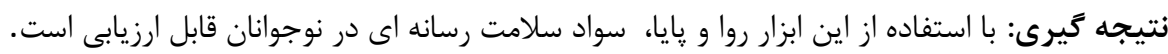




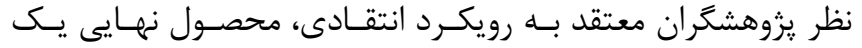

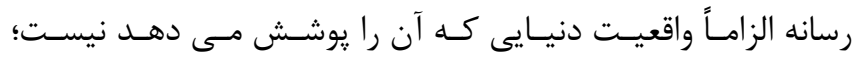

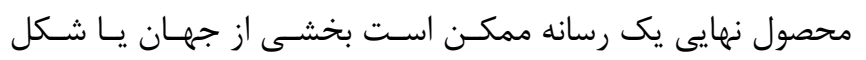

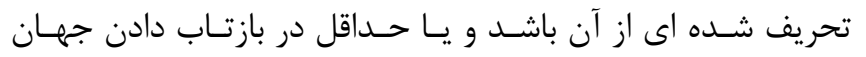

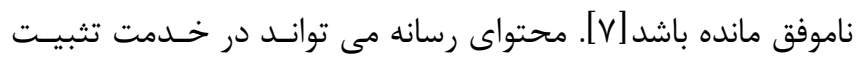

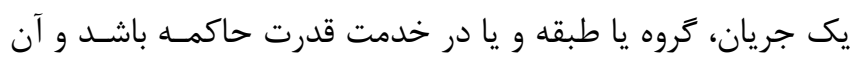

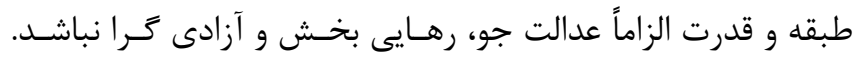

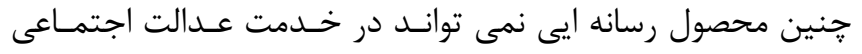

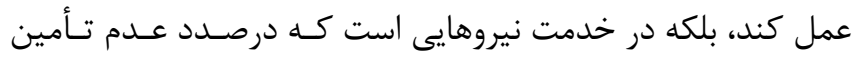

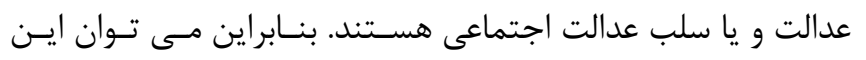

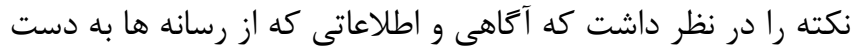

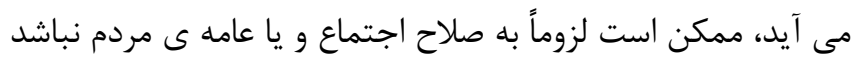

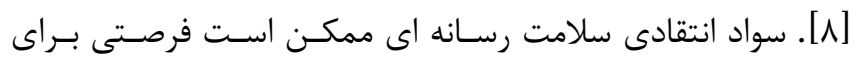

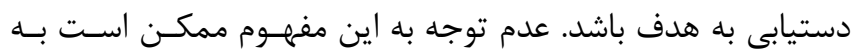

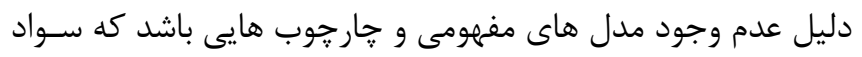

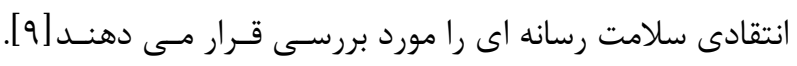

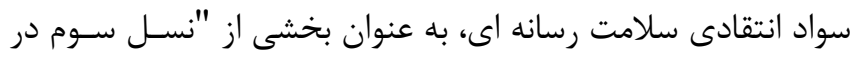

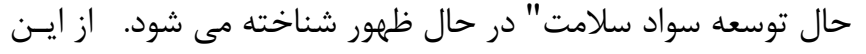

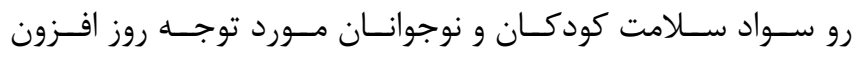

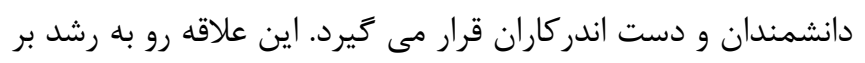

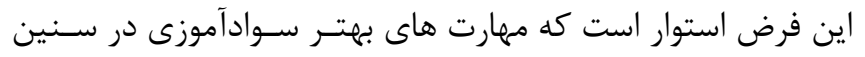

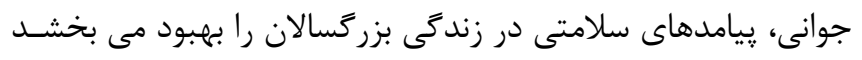

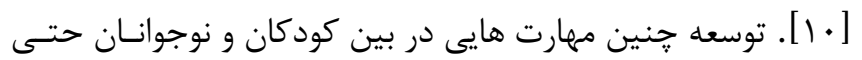

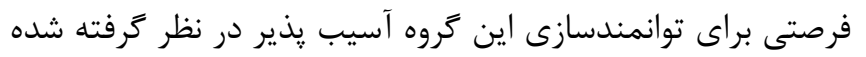

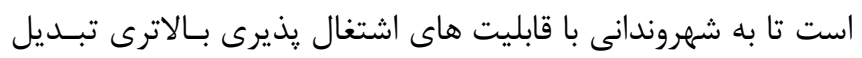

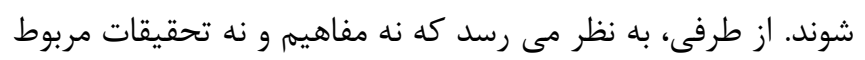

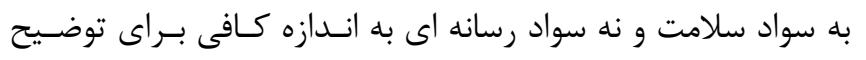

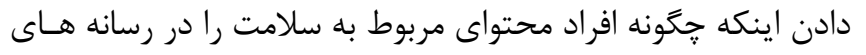

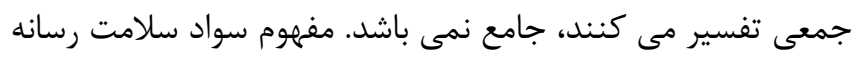

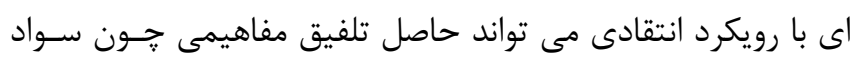

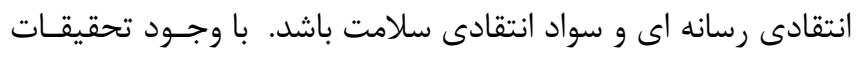

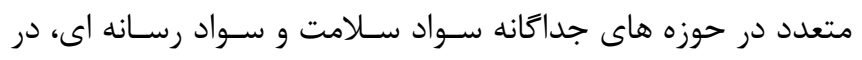

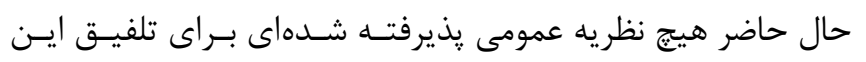

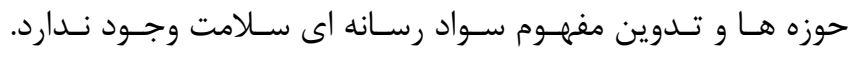

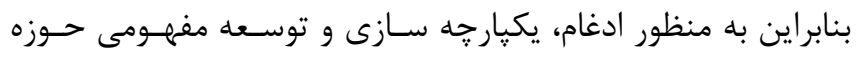

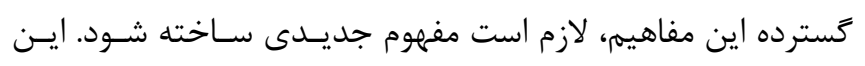

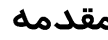

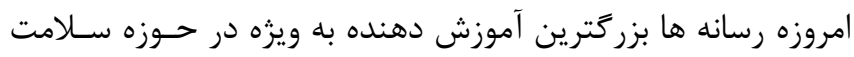

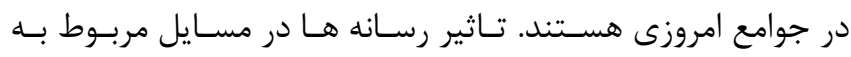

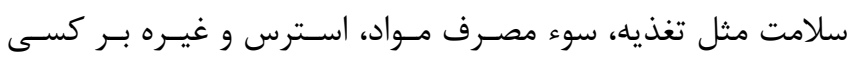

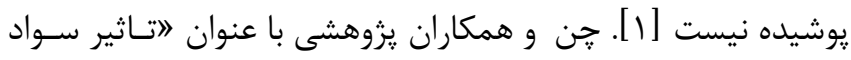

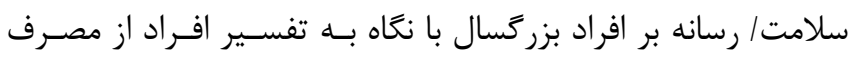

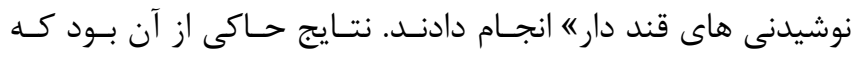

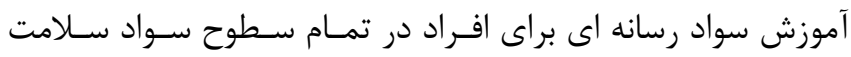

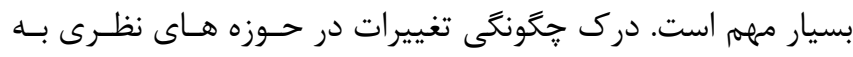

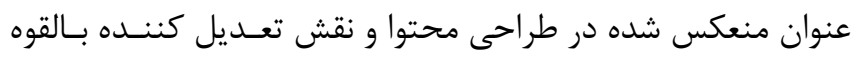

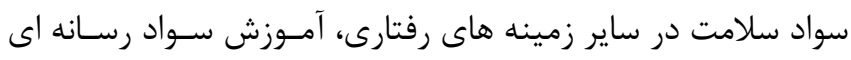

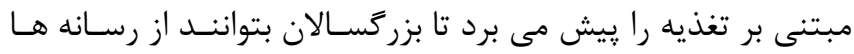
ييشى بكيرند [r]

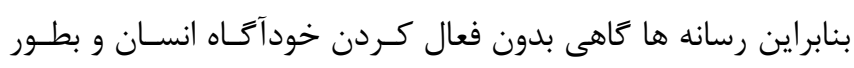

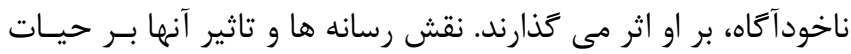

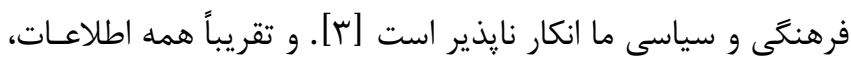

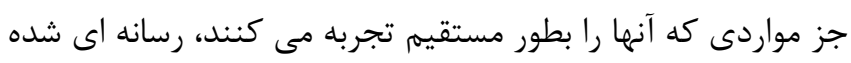

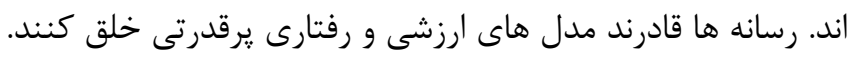

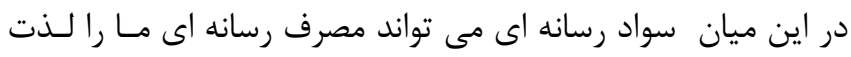

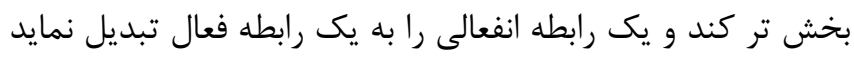

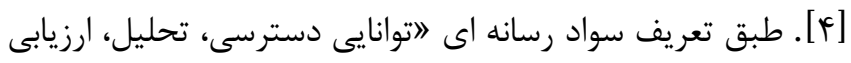

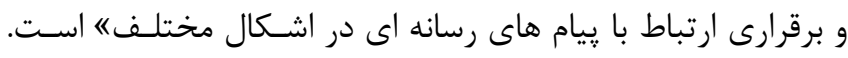
اين كونه از سواد؛ با توانمند كردن انسان بر درك نحساط نحوه كار رسانه ها

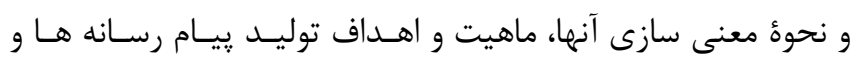

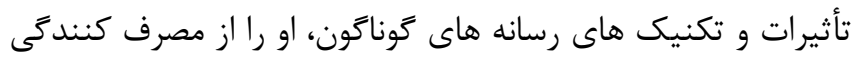

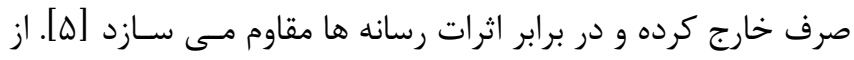

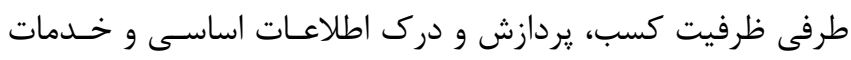

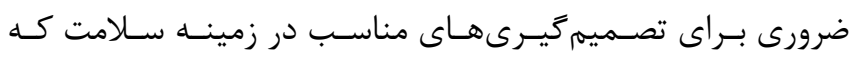

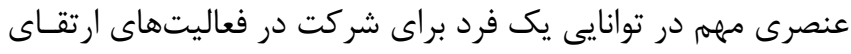

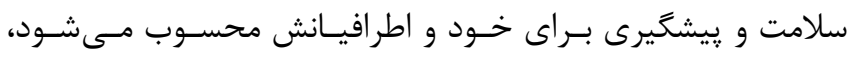

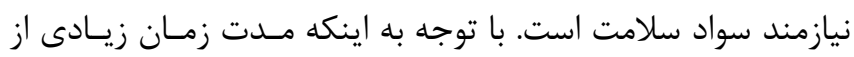
وقت افراد صرف مشاهده رسانه ها مى شود، اين رسانه ها مى توانـــ

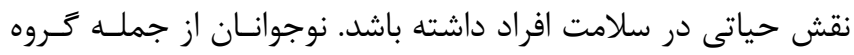

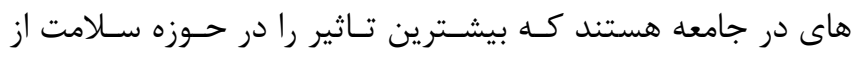

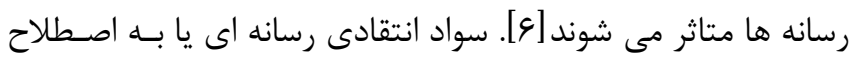

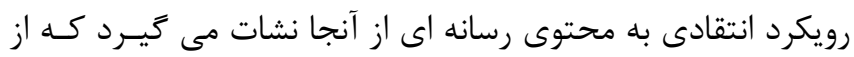


نيازمند تغييراتى اساسـى در راهبردهـاى حـوزه انتقــادى هسـتند.

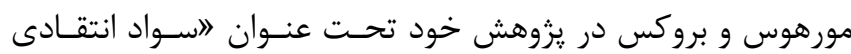

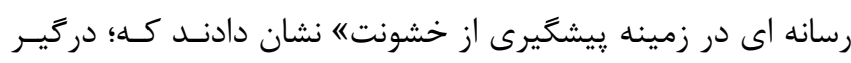

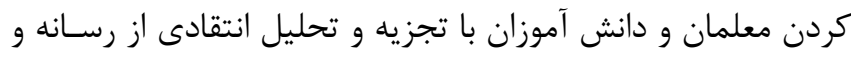

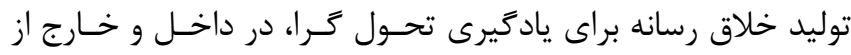

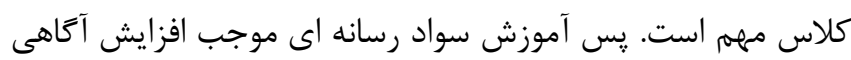

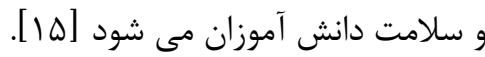

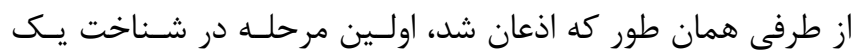

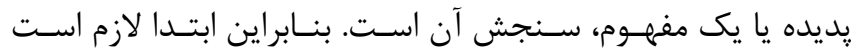

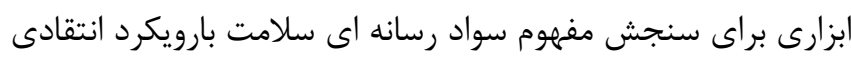

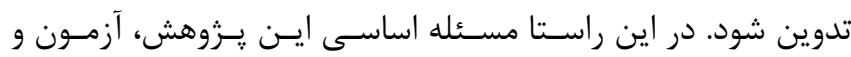
اعتباريابى ابزار سواد سلامت رسانه اى با رويكرد انتقادى براى دانش

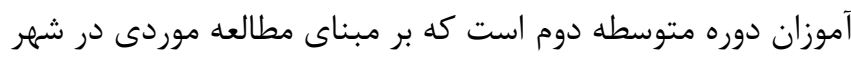

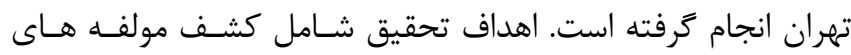

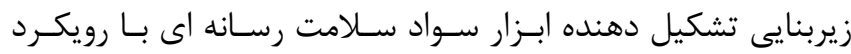
انتقادى و اعتباريابى ساختار عاملى ابزار مذكور است.

\section{مواد و روش كار}

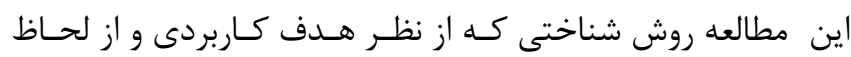

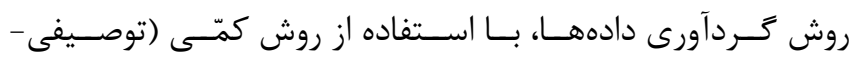

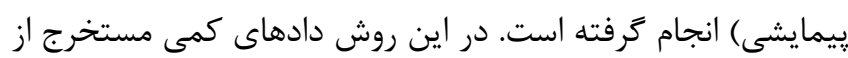

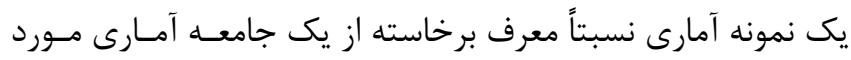

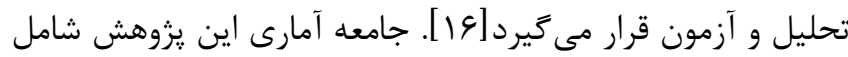

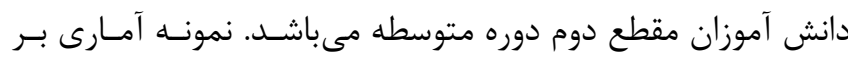

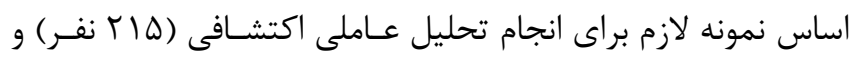

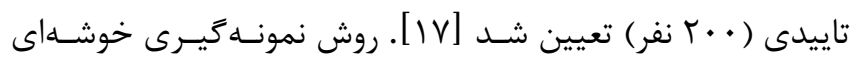
متناسب با حجم خوشه بود، بدين صورت كه ابزار محقق سـاخته در

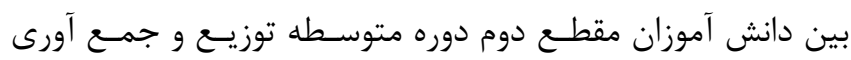

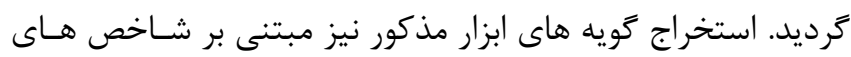

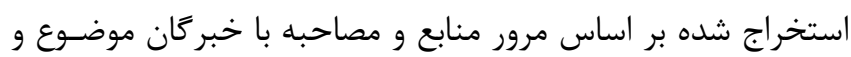

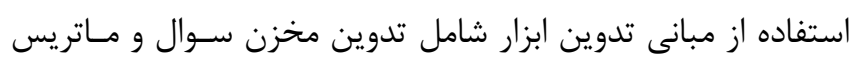

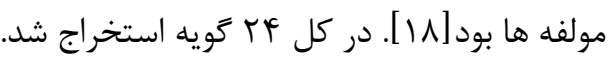

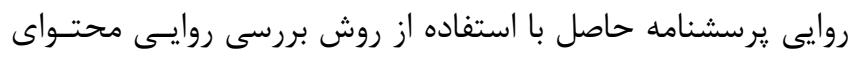
كمى يعنى نسبت روايى محتوايى (CVR) و شاخص روايى محتوايى

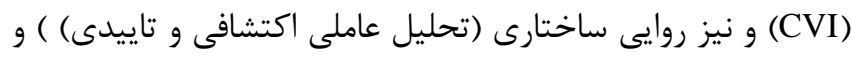

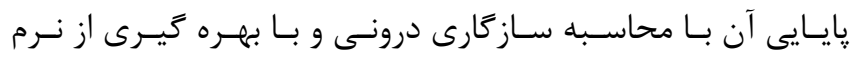

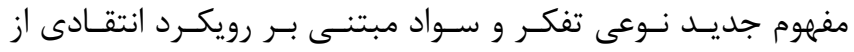

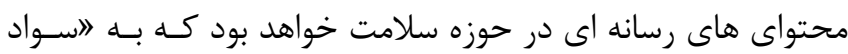

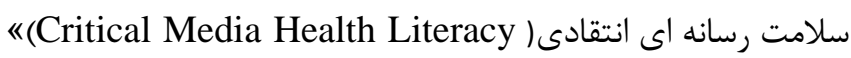

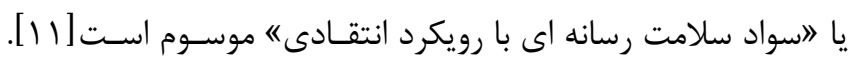

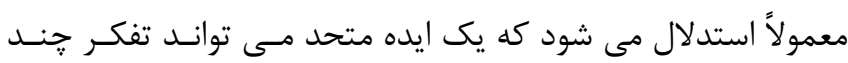

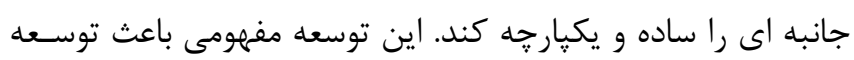

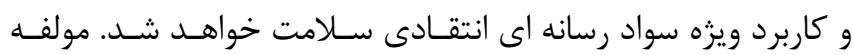

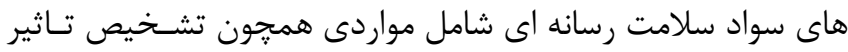

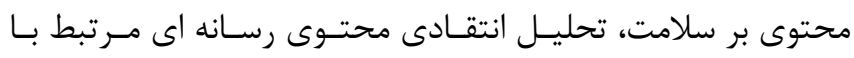

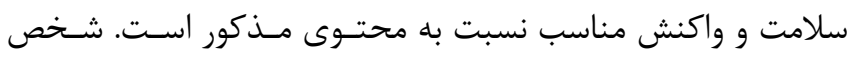

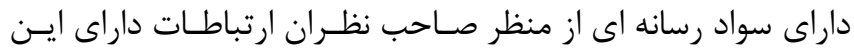

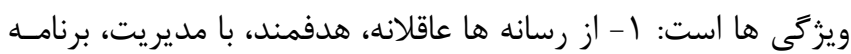

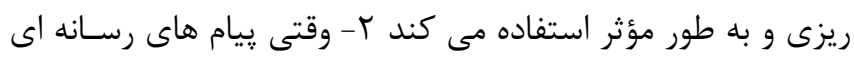

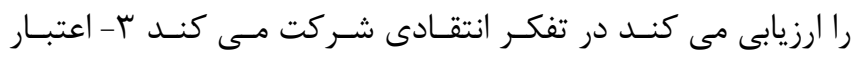

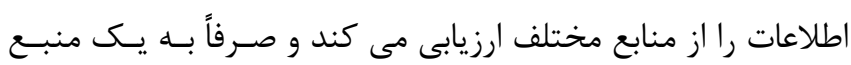

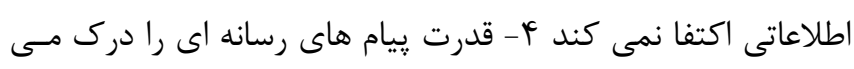

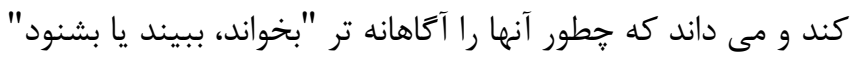

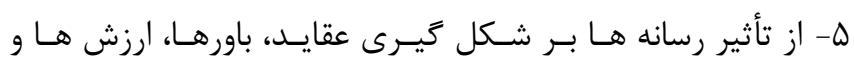

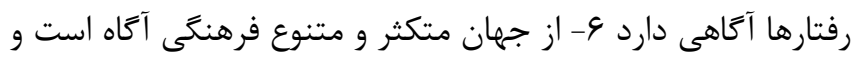

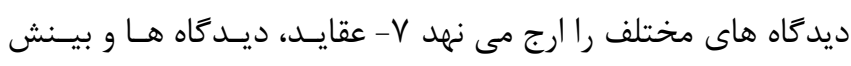

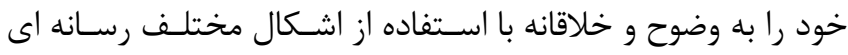

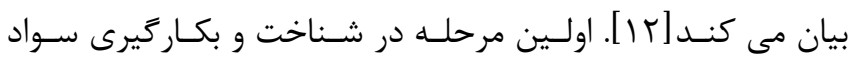

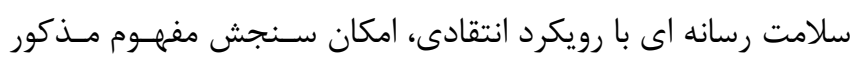

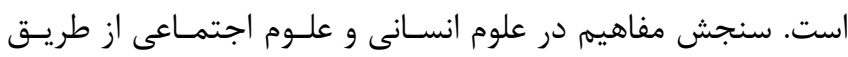

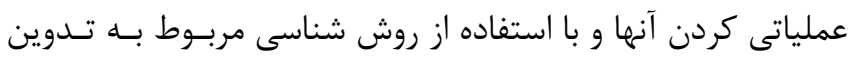

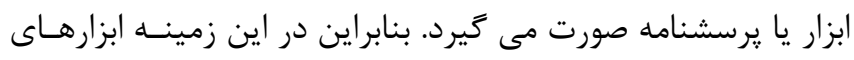
سنجش تدوين و توسعه קيدا كردند.

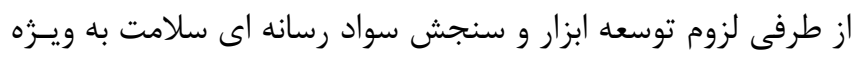

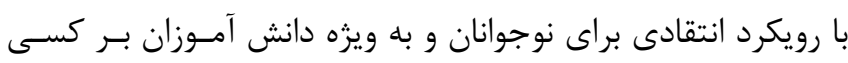

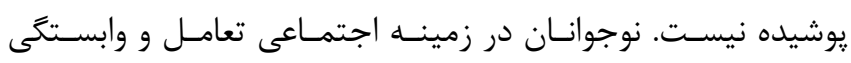

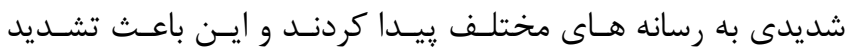

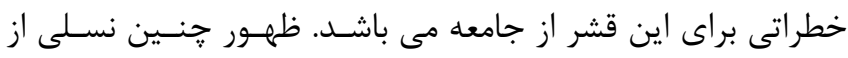

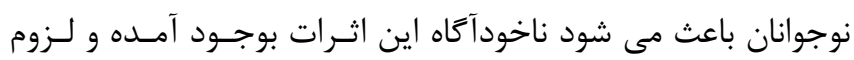

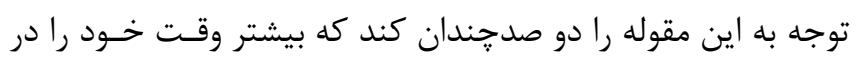

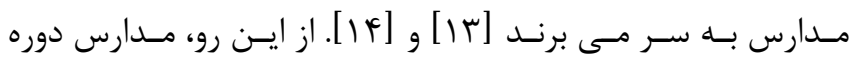

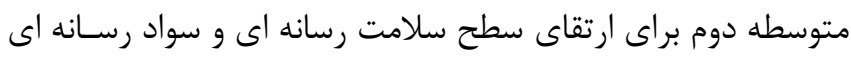


سال بيستم، شماره هنجم، مهر - آبان ••lf

شود و در نهايت تعداد سوالات باقيمانده ابزار سـواد سـلامت رســانه

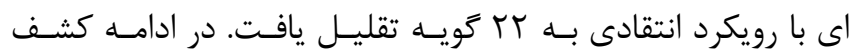

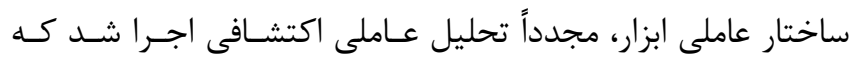

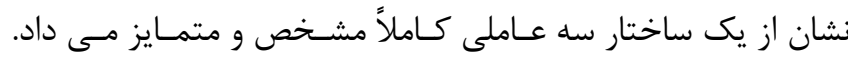
مقدار آماره KMO براى كفايت نمونه كيرى مقدار • Mr/ • بـوده و

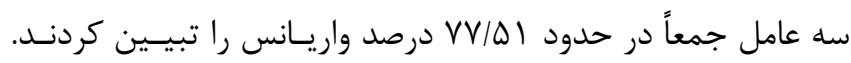

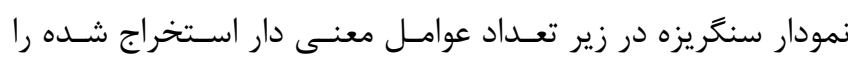

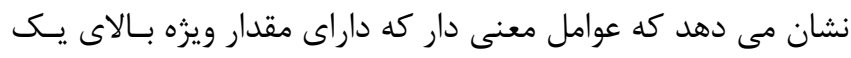

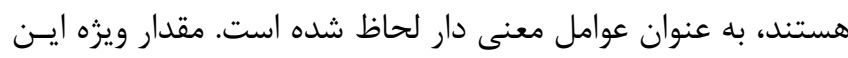
سه عامل در شكل آمده آمد است.

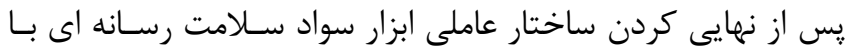

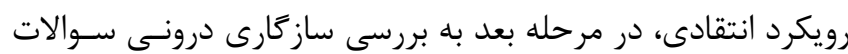

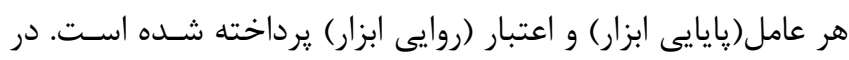

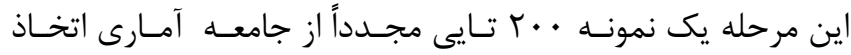

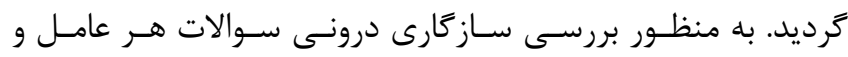

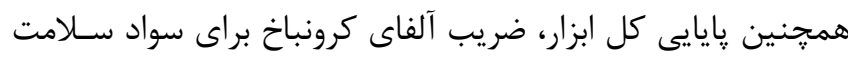

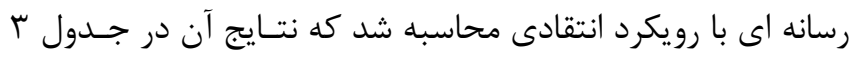

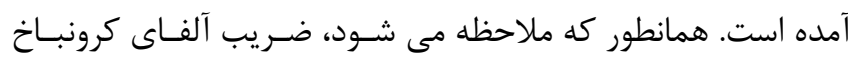

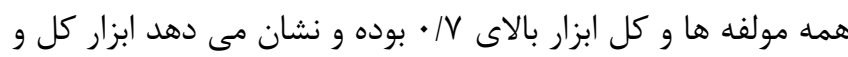

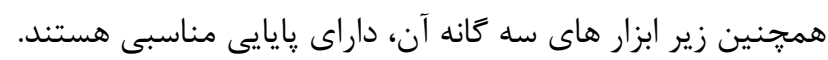

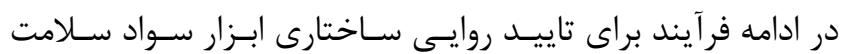

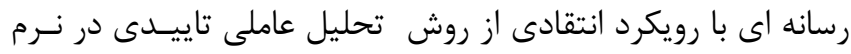

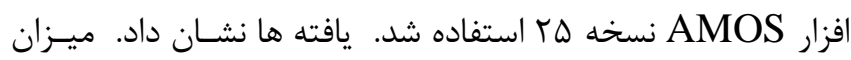

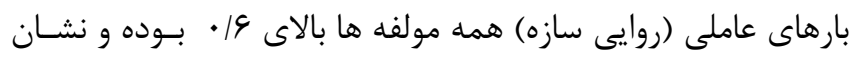

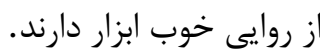

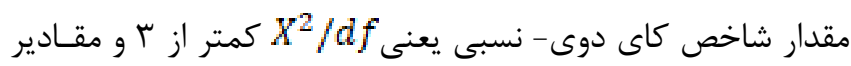

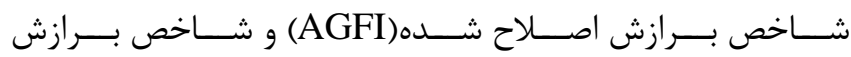

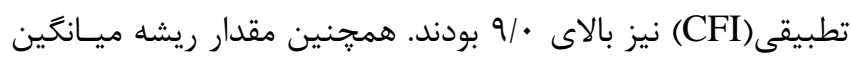

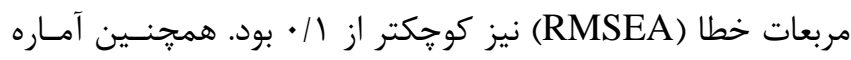

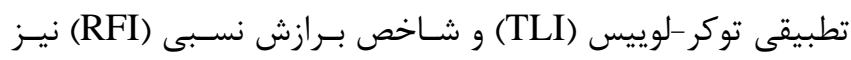
بالاتر از 9/• بودند. آماره شاخص هولتر براى مقدار نمونه بحرانى نيز

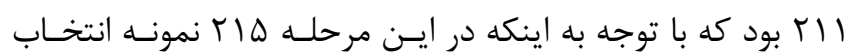

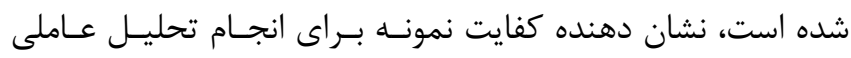

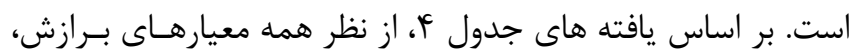

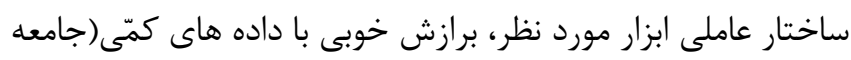

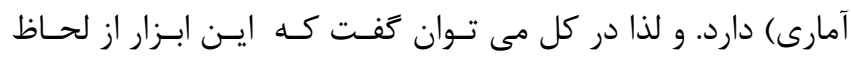

افزارهاى SPSS و AMOS نسـخه فT بر رسى شـد. بــه منظـور

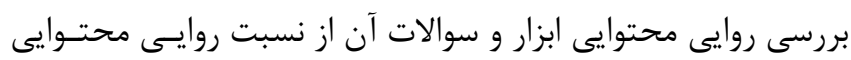

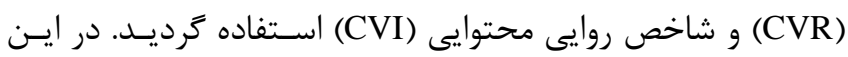

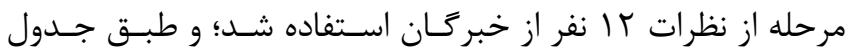

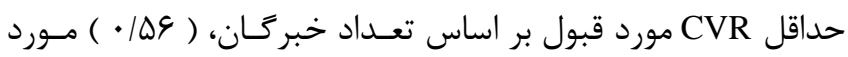

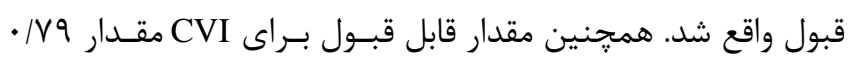

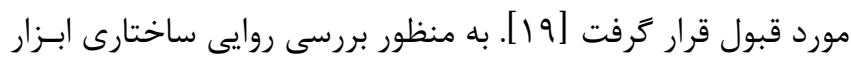

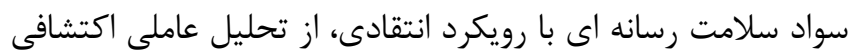

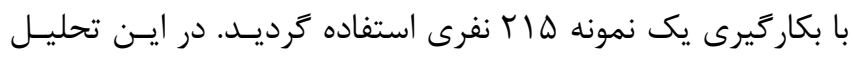

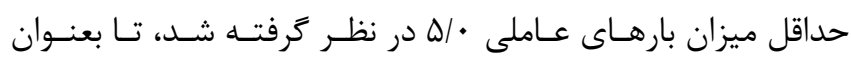

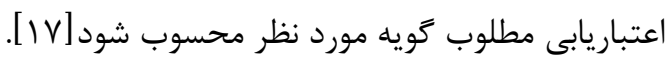

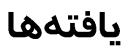

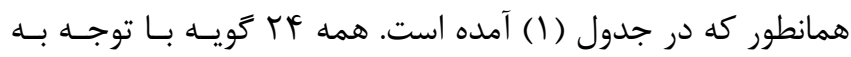

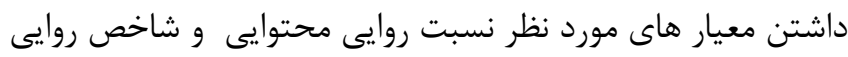

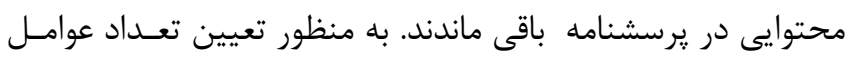
(مولفه هاى) استخراج شده توسط تحليل عاملى اكتشافى و مقــادير

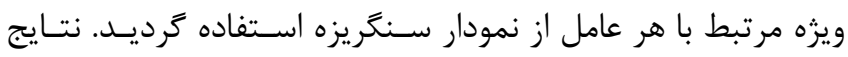

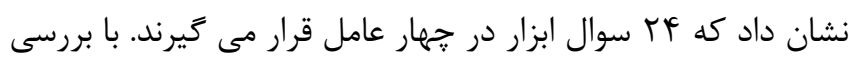

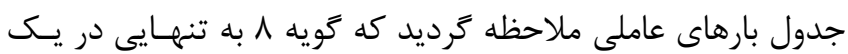
عامل قرار مى كيرد. متن كويه به صورت زير بود: "وقتى مطلبى درد

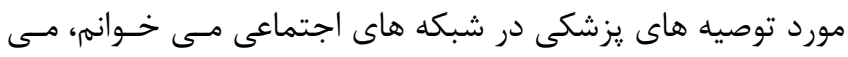

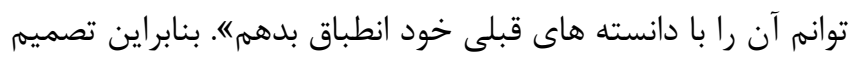

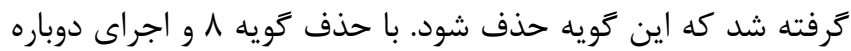

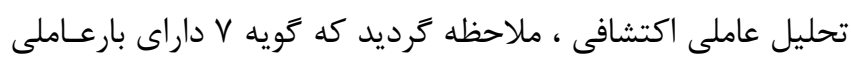

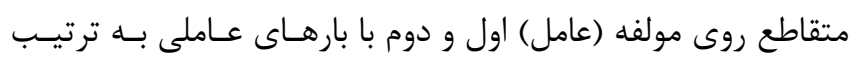

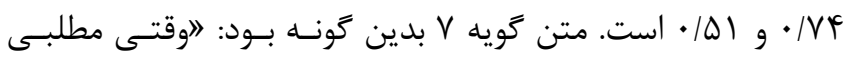

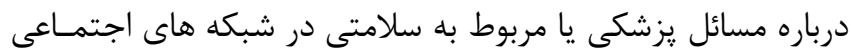

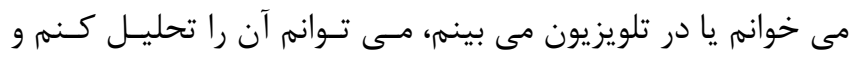

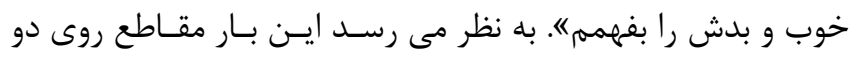

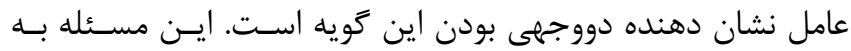

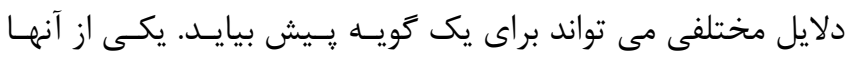

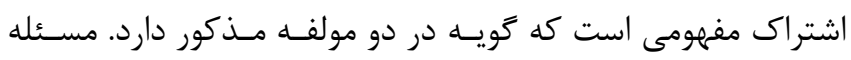

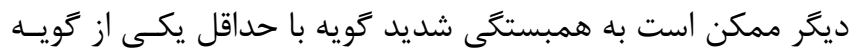

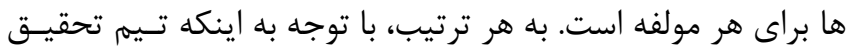

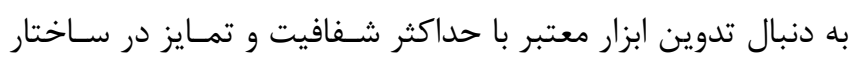

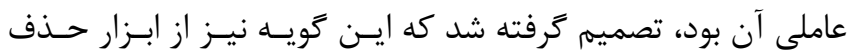


نشريه يزوهشكده علوم بهداشتى جهاددانشكاهى

سوالات ابزار يا مولفه هاى آن) و n تعداد سوالات براى كل ابـزار يــاـ

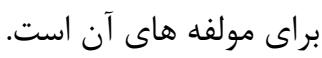

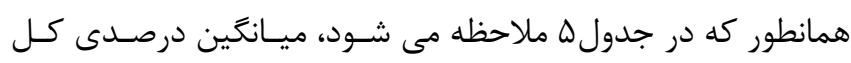

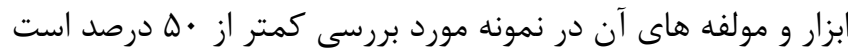
و نشان مى دهد كه ميزان سواد سلامت رسانه ایى در نمونه مـذكور

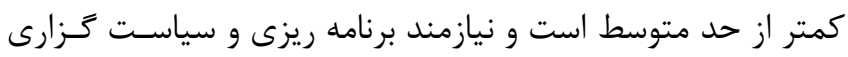
نهادهاى مربوطه براى ارتقاى سطح سواد رسانه اى سلامت است.
اعتبار (روايى) از تبيين ( برازش) خوبى با داده هاى جامعـه آمـارى

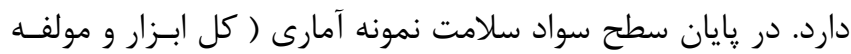

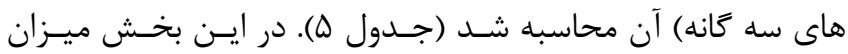

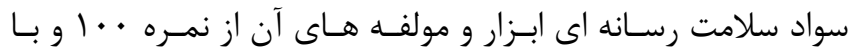
استفاده از فرمول زير محاسبه شد:

$$
P=\frac{S}{n} \times 20
$$

در اين فرمـول P نمـره درصـدى و S نمـره خـام (مجمـوع نمـرات

\begin{tabular}{|c|c|c|}
\hline CVR & CVI & \\
\hline$\cdot / N Y$ & $\cdot / \wedge \oint$ & Q1 \\
\hline$\cdot / \Lambda 9$ & . $/ 19$ & Q2 \\
\hline 1 & $\cdot / \Lambda q$ & Q3 \\
\hline • NYr & $\cdot|\Lambda|$ & Q4 \\
\hline .191 & $\cdot|\Lambda|$ & Q5 \\
\hline . NTr & $\cdot \mid \Delta r$ & Q6 \\
\hline$\cdot / \wedge 9$ & . 194 & Q7 \\
\hline 1 & 1 & Q8 \\
\hline$\cdot \mid \wedge \varphi$ & - IA & Q9 \\
\hline$\cdot / \wedge 9$ & $\cdot / 19$ & Q10 \\
\hline$\cdot / \wedge 9$ & $\cdot / 19$ & Q11 \\
\hline 1 & . & Q12 \\
\hline . NTr & $\cdot|\Lambda|$ & Q13 \\
\hline$\cdot \mid \wedge \varphi$ & $\cdot|\Lambda|$ & Q14 \\
\hline 1 & 1 & Q15 \\
\hline$\cdot / \wedge 9$ & $\cdot / 19$ & Q16 \\
\hline • & . & Q17 \\
\hline 1 & . & Q18 \\
\hline . NT & $\cdot|\Lambda|$ & Q19 \\
\hline$\cdot / \Lambda$ & $\cdot \mid 19$ & Q20 \\
\hline . & 1 & Q21 \\
\hline$\cdot / \Lambda 9$ & - & Q22 \\
\hline
\end{tabular}




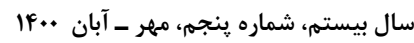

جدول ז: ماتريس بارهاى عاملى خرخش يافته ابزار سواد سلامت رسانه اى با رويكرد انتقادى

\begin{tabular}{|c|c|c|c|c|}
\hline 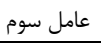 & عامل دوم & عامل اول & شماره سوال & \\
\hline - /TAK & $\cdot / 4 \mid \lambda$ & - /ADT & 1 & \multirow{22}{*}{ سواد سلامت رسانه اى با رويكرد انتقادى } \\
\hline - & $\cdot|4| \lambda$ & - /ADT & $r$ & \\
\hline$\cdot / / \Lambda$. &. $\mid 411$ & - MAFT & r & \\
\hline . &.$/ F r$. & - /ATA & k & \\
\hline 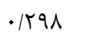 & $\cdot \mu \cdot r$ & |Arr & $\Delta$ & \\
\hline$\cdot / r \cdot 4$ & $\cdot / \pi r$. & ./VVT & 4 & \\
\hline . /TTS &.$\pi s 9$ & - V V D & v & \\
\hline$\cdot / \cdot \wedge \Lambda$ &.$/ \cdot \mu q$ & $\cdot / \mathrm{rr}$. & $\wedge$ & \\
\hline . KFA & .1111 &.$/ q \Delta F$ & 9 & \\
\hline$\cdot / l V t$ & . IANT & ./lFF & 1. & \\
\hline$\cdot / 1 \Delta \Lambda$ & . IAVQ & $\cdot / 499$ & 11 & \\
\hline.$/ \cdot r F$ & $\cdot|\wedge \&|$ &.$/ 191$ & ir & \\
\hline .1 .94 & . ATI & $\cdot / r \cdot 9$ & r & \\
\hline.$/ 19 \Lambda$ & . $/ A \mid a$ & $\cdot \pi+q$ & if & \\
\hline$\cdot|\cdot+|$ & .1919 & $\cdot / r \cdot$ & 10 & \\
\hline.$/ 1 \Delta V$ & $\cdot 19 \cdot V$ & - MFFT & 19 & \\
\hline$\cdot / 110$ & $\cdot / 1 \mathrm{r}$ & $\cdot / \pi \mid \cdot$ & iv & \\
\hline$\cdot 11 \cdot 9$ & $\cdot \pi \omega$ & .441 & 11 & \\
\hline$\cdot 11 \cdot 9$ & $\cdot \pi \Delta$ & . & 19 & \\
\hline •/マ१९ & $\cdot 1 \cdot v 9$ &.$/ 4 I V$ & $r \cdot$ & \\
\hline . /Vaf & $.1 .1 r$ & .1191 & rl & \\
\hline . IVAY & r.|. & ./.r. & Tr & \\
\hline
\end{tabular}

جدول ؟ّ: ضرايب آلفاى كرونباخ و يايايى تركيبى براى تك تك مولفه هاى الكَوى برازش شده

\begin{tabular}{|c|c|c|}
\hline يايايى كل ابزار & ضريب آلفاى كرونباخ & \\
\hline \multirow{3}{*}{ - /VAF } &.$/ 94 \wedge$ & تشخيص تأثير محتواى رسانه بر سلامت \\
\hline & . /9Tt & تحليل انتقادى محتواى مرتبط با سلامت در رسانه \\
\hline &.$/ 91 \mathrm{~V}$ & واكنش نسبت به محتواى رسانه \\
\hline
\end{tabular}

جدول F: شاخص هاى نيكويى برازش ابزار سواد سلامت رسانه اى با رويكرد انتقادى

\begin{tabular}{|c|c|}
\hline بر آورد آماره & \\
\hline $4 / L$ & نسبت مجذور خى به درجه آزادى(CMIN/DF) \\
\hline$\cdot 1 \cdot 4$ & شاخص ريشه دوم ميانكين مربعات باقيمانده(RMSEA) \\
\hline.$/ 91$ & شاخص نكويى برازش تعديل شده(AGFI) \\
\hline • / वा & شاخص برازش تطبيقى(CFI) \\
\hline.$/ 9$ & شاخص برازش توكر-لويس(TLL) \\
\hline.$/ 95$ & شاخص برازش نسبى(RFI) \\
\hline
\end{tabular}

جدول ه: ميانكَين درصدى كل و مولفه هاى ابزار سواد سلامت رسانه اي با رويكرد انتقادى

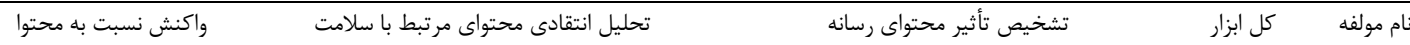




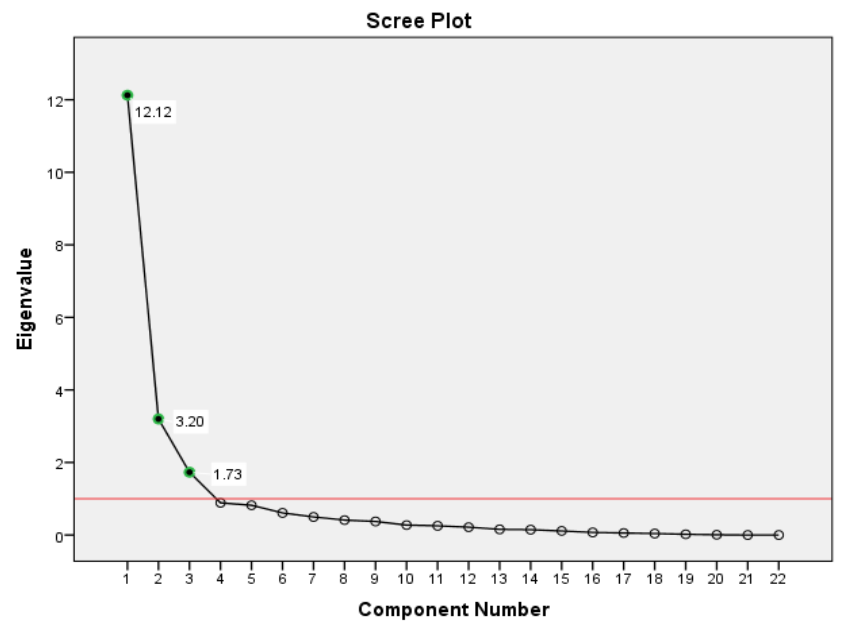

شكل ا: نمودار سنكرَيزه (برش) براى تعيين تعداد زيرابزار هاى معنى دار استخراج شده

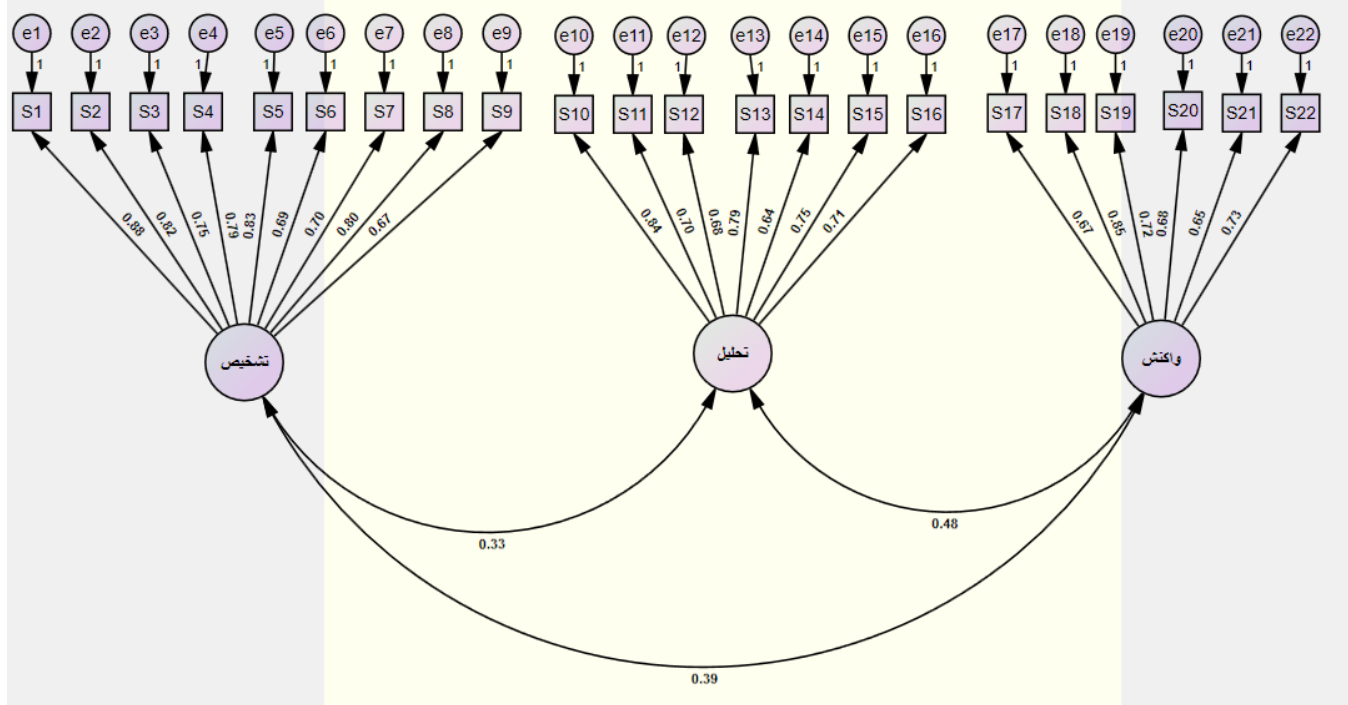

شكل r : نمودار خروجى تحليل عاملى در نرم افزار Amos

انتقادى نسبت به محتواى رسانهه مى باشـد. نتـايج تحليـل عـاملى

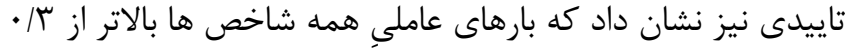

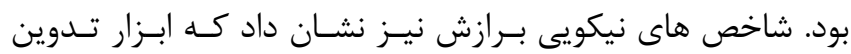

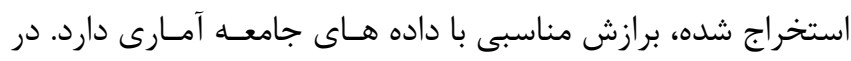

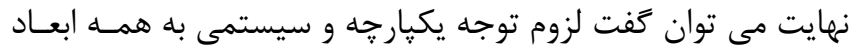

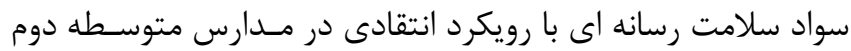

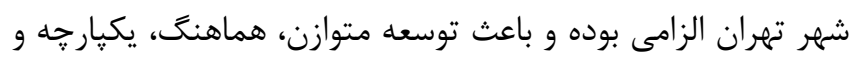
اثربخش سلامت رسانه اى در مدارس مذكور است تـا بتـوان شـاهد

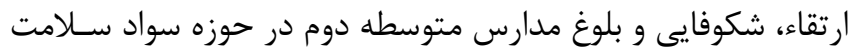

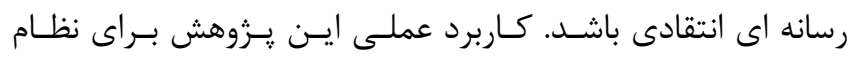




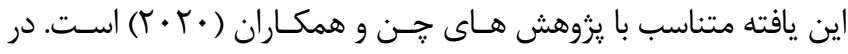

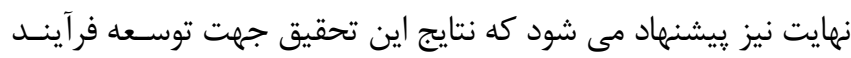

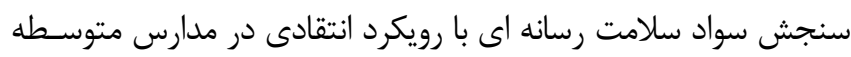

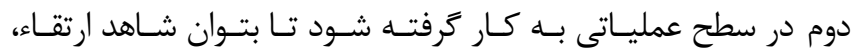

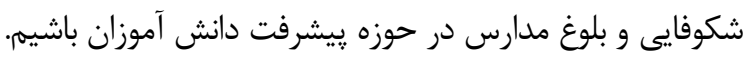

$$
\begin{aligned}
& \text { سهم نويسند كان } \\
& \text { ابوالقاسم رئيسى نافجى: طراحى و اجرا مطالعه و نغارش مقاله }
\end{aligned}
$$

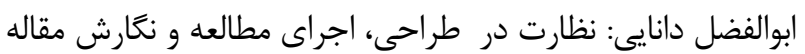

$$
\begin{aligned}
& \text { محمد زركر: مشاركت در طراحى مطالعه و نتارش مقاله }
\end{aligned}
$$

\section{منابع}

1. Labas D, Males D. Adolescent perception of electronic media ethical values in the context of sociode-mographic characteristics and media literacy. Nova Prisutnost 2017; 15: 211-230

2. Chen Y, Porter KJ, You W, Estabrooks P, Zoellner JM. A health/media literacy intervention improves adults' interpretations of sugar-sweetened beverage advertising. Journal of Media Literacy Education 2020; 12: $70-83$

3. De Leeuw E. The Political Ecosystems of health literacies .Health Promotion International 2012; 27: 1-4 4. Domanska A. Do adolescents understand the items of the European Health Literacy Survey Questionnaire (HLS-EU-Q47)-German version? Findings from cognitive interviews of the project "Measurement of Health Literacy among Adolescents" (MOHLAA) in Germany. Archives of Public Health 2018; 76:7-12

5. Jafari A, Peyman N. Application of theories/models of health education and promotion in Health Literacy research: a systematic review. Journal of Health Literacy 2018; 3: 124-136 [In Persian]

6. Nasiri B, Bakhshi B, Hashemi M. The importance of media literacy education in the 21st century. Media Studies 2012; 1: 149-158 [In Persian]

7. Driessnack M, Chung S, Perkhounkova E, Hein M. Using the "newest vital sign" to assess health literacy in children. The Journal of Pediatric Health Care 2014; 28 : 165-71

8. Tehrani H. Media Health Literacy. Journal of Health Literacy 2016; 1: 141-146. [In Persian]

9. Chinn D. Critical Health Literacy: a Review and Critical Analysis. Social Science and Medicine 2011; 73: 60-67

10. Higgins JW, Begoray D. Exploring the Borderlands between Media and Health: Conceptualizing 'Critical

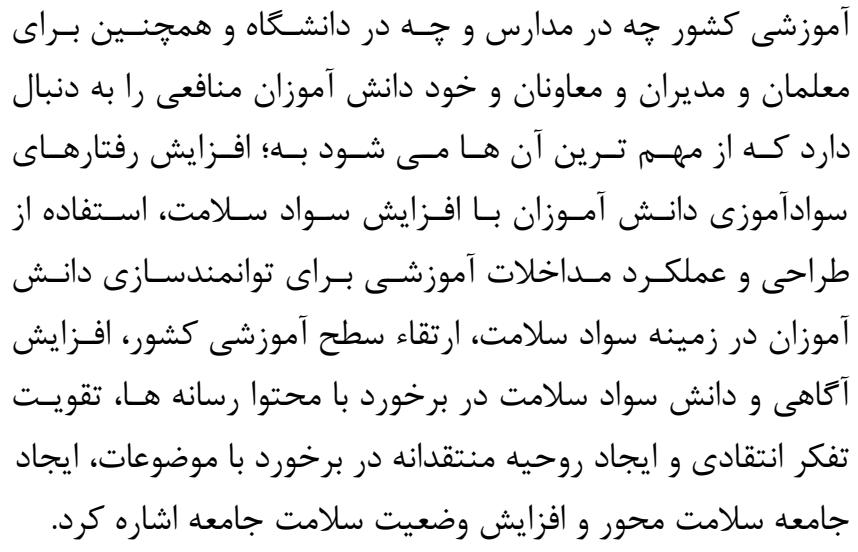

Media Health Literacy'. Journal of Media Literacy Education 2012; 4: 136-148

11. Berkman N, Davis $\mathrm{T}$, McCormack L. Health literacy: what is it? Journal of Health Communication 2010; 15: 9-19

12. Khodabandeh M, Maleki Avarasin S, Nikniaz L. The Relationship between Health Literacy, Perceived self-efficacy and Self-care Performance of Female Senior High School Students in Health Promoting Schools of Miyaneh, 2016-2017. Journal of Health Literacy 2017; 2: 164-176 [In Persian]

13. Qadian Anarmarzi F. The role of media literacy on women's health literacy in cyberspace use. Journal of Applied Studies in Social Sciences and Sociology 2019; 2: 1-12 [In Persian]

14. Waezi Mm Niknami Sh, Haidarnia A, Goodarzi A. The effect of iron deficiency anemia media education package on health literacy of high school girls in Chabahar city. Military Surveillance Sciences 2018; 5: 156-162 [In Persian]

15. Dehghankar L, Panahi R, Yekefallah L, Hosseini N, Hasannia E. The study of health literacy and its related factors among female students at high schools in Qazvin. Journal of Health Literacy 2019; 4: 18-26 [In Persian]

16. Sarmad Z, bazargan A, Hejazi A. Research Methods in Behavioral Sciences. $1^{\text {st }}$ Edition Samat Publications: Tehran, 2020 [In Persian]

17. Klein p. An easy guide to factor analysis. Translated by Seyyed Jalaluddin Sadr Sadat and Asghar Minaei. $1^{\text {st }}$ Edition Samat Publications: Tehran, 2016 [In Persian]

18. Farasatkhah M. Qualitative research methodology in social sciences with emphasis on basic theory (Grounded Theory, GMT). Tehran, $1^{\text {st }}$ Edition, Agah Publications: Tehran, 2016 [In Persian] 